\title{
Factors associated with heart failure readmissions from skilled nursing facilities
}

Background: Despite guideline-driven pharmacological therapies and careful transitional care, the rates of preventable hospital re-admission of heart failure patients and associated costs remain unacceptably high in the SNF populations. Transfer to SNF is one strategy to limit hospitalizations. As such, $25 \%$ of patients are still symptomatic at time of discharge.

Purpose: The objective of this study is to identify patient factors affecting re-admissions of HF patients residing in SNF within 30-days.

Methods: A retrospective electronic chart review was completed on patients $>65$ years with HF who were admitted into large medical center between 2012 and 2014. Descriptive statistics and univariate analyses using the chi-square test or Fisher's exact test for categorical variables and the Mann-Whitney test for continuous data was used to compare patients readmitted within 30 days $v s$. those who were not readmitted within 30 days. Significant factors associated with readmission in the univariate analysis $(p<0.10)$ were included for a multivariate logistic regression model. The analysis of LOS was accomplished by applying standard methods of survival analysis, i.e., computing the Kaplan-Meier product limit curves, where the data were stratified by readmission within 30 days (Yes vs. No). No data were considered 'censored'. The groups were compared using the log-rank test. The median rates for each group were obtained from the Kaplan-Meier/Product-Limit Estimates and their corresponding $95 \%$ confidence intervals were computed, using Greenwood's formula to calculate the standard error. Unless otherwise specified, a result was considered statistically significant at the $p<0.05$ level of significance.

Results: Fifteen variables: creatinine, weight difference, CKD, Angina, Arrhythmia, VHD, Tobacco, ADL, independent in bathing, independent in the toilet, S3 Heart sounds present, $\mathrm{HJR}, \mathrm{AF}$, Nitrates, and Hydralazine, were identified for the multivariate logistic regression as potential risk factors associated with "readmission within 30 days". Based on 23 readmissions within 30 days, our final model included only 2 predictor variables. Creatinine and ADLs were included in the final model as this subset of predictors was found to be the best for prediction of "readmission within 30 days". Creatinine $(p<0.0087)$ and ADLs $(p<0.0077)$ were both significantly associated with readmission within 30 days in the final logistic regression model. Every 1-unit increase in creatinine was associated with an $87 \%$ increase in the odds of being readmitted within 30 days $(O R=1.87)$. Those patients who require assistance with ADLs were over 9 times more likely to be readmitted within 30 days $(O R=9.25)$ as compared to patients who are independent.

Keywords: Heart failure; Re-admissions; Skilled nursing facilities (SNF); Nursing homes (NH); Factors
Shade Akande*

Stony Brook University, NY, USA ${ }^{*}$ Author for correspondence: fjadesola@aol.com

Submitted: 07 December 2016 Accepted: 15 December 2016 Published online: 20 December 2016
Heart failure (HF) affects more than 5 million patients in the United States and by 2030, > 8 million people in the United States (1 in every 33) will have HF [1]. Annually, more than 1 million patients are hospitalized with a primary diagnosis of heart failure, accounting for a total Medicare expenditure exceeding \$17 billion with $>50 \%$ patients readmitted to hospital within 6months of discharge. Annual expenditures for both primary and secondary diagnosis of heart failure have been estimated to be as high as $\$ 38$ billion of which $\$ 23$ billion is for hospital stay. Between 2012 and 2030, total direct medical costs of $\mathrm{HF}$ are projected to 
increase from $\$ 21$ billion to $\$ 53$ billion [2]. The rates of readmissions to the hospital for HF are high and expected to increase with an estimated yearly incidence of 550,000 readmissions and 658,000 annual ED encounters [3].

The financial implications of readmission are significant. Advanced heart failure is characterized by progressive clinical deterioration reflected in frequent hospital admissions and rising costs. In order to decrease costs, the National Quality Forum has endorsed hospital risk-standardized readmission rates (RSRRs) as performance measures [3]. The patient protection Affordable Care Act 2010 created incentives to reduce readmission, with penalties for hospitals which included zero reimbursement for services provided and/or loss of revenue if the HF patient is readmitted within 30 days [4]. Hospitals with high readmission rates may be subject to lose $-3 \%$ of their Medicare reimbursement by 2015 [3].

In order to reduce readmissions, patients may be referred to skilled nursing facilities (SNF) and Nursing Homes $(\mathrm{NH})$ after hospitalization for recuperative care and to maximize pharmacological therapies. This has increased to serve as a potential strategy for reducing readmissions [5]. However, in spite of the pharmacological and transitional care from hospital to SNFs, the rates of preventable re-admission remain unacceptably high.

Reducing HF hospital readmission rates from SNF and $\mathrm{NH}$ has become a national priority because approximately $20 \%$ of these patients are Medicare beneficiaries and are readmitted within 30 days [4]. Readmissions are multifactorial and problematic in this unique population because of high cost, and disruptions in care, disease progression, and increased mortality. HF readmissions are a complex problem and a single solution has not been sufficient in decreasing the readmission risk. Identification of heart failure predictors in patients from SNFs and NH may lead to an improved referral pattern and an improved 30 day outcome [6,7].

The objective of this study is to identify patient specific factors affecting readmissions of HF patients from SNFs within 30-days.

\section{Literature Review}

Almost one quarter of older hospitalized adults with HF are discharged to SNFs [8]. Nearly half of the 164,672 patients with HF that were discharged to SNFs, were readmitted to a hospital within 90 days after discharge, and 30\% died within 90 days [7]. Heart Failure is a heterogeneous condition, with many causes. Accurate classification of patient factors that affect readmissions may have positive impact on outcomes of patients that resides in SNFs.
Many factors may contribute to readmission of $\mathrm{HF}$ patients. These factors include comorbidities such as diabetes and decreased renal function at discharge [9]. Other factors influencing readmission in HF patients include dyspnea, increasing age, renal problems, fluid status/weight, anemia, LOS, ADLs, BNP, hypotension, and comorbidities. The proportion of patient factors responsible for avoidable readmissions varies extensively between studies.

Numerous studies have identified dyspnea as the strongest predictor of readmission [10-16]. Up to $77 \%$ of patients who were admitted for acute episodes of heart failure initially presented to the emergency department with dyspnea [13]. A majority of patients who require re-admission for HF have shortness of breath. Patients are dyspneic with exertion and at rest. Fatigue is also present in this group of patients. However, dyspnea is the primary factor for seeking care by patients and for referral of patients to hospitals for admission [13]. Ninety three percent of the SNFs population studied reports dyspnea as breathing related problems that predicts re-admission [10].

Timeliness in reporting of symptoms of dyspnea has been associated with decreased risk of re-admission. Researchers found a correlation between relieving of dyspnea and decreased risk of 30 day re-admission in 45\% HF patients [14]. Breathing related problems reported by $93 \%$ of $\mathrm{HF}$ patients led to them seeking care early and thereby preventing re-admission [11]. A delay in reporting symptoms and seeking care, which can range from 6 hours to 3 days, appears to correlate with deterioration and re-admission [17]. Studies of symptom monitoring and response training appeared to have an early but not sustained benefit resulting in no difference in 90-day event-free survival [18].

Consistently, researchers report increasing age as one of the many factors that accounts for avoidable readmissions. This non-modifiable risk factor is often associated with cognitive deficits. Older age, disability status and increased LOS were associated with readmission [19]. Social and environmental factors may influence the severity and adaptability of aging process in older HF patients. Advanced age has been implicated as predictor of re-admissions [20-22]. In 161 elderly patients that had four or more $\mathrm{HF}$ admissions, it was found that one of the predictors of readmission was increasing age [23].

Advanced age has also been identified as a cause of frequent re-admissions in the SNFs population. The re-admission rate was increased from $23 \%$ to $27 \%$ within 30 days with increased age [10]. In addition, advanced age was identified as a significant contributor to re-admission among US veterans residing at SNFs [19].

Worsening renal function which is shown as an increase in BUN/Creatinine is a strong predictor of re- 
admission [10,14,24-30]. Improved assessment of $\mathrm{BUN} / \mathrm{Cr}$ may lower hospital re-admission rates. However, the management of HF may precipitate decreased renal function because of the use of ACE inhibitors and diuretics that are often prescribed for HF patients. Guideline-driven therapies include ACEi optimization and neuro-hormonal antagonists, which may further worsen the BUN/Cr and lower the blood pressure. This can lead to hypotension and worsening of the renal function, and diuretics that are often administered for symptoms relief of dyspnea may leads to hyponatremia [5].

Fluid volume status or weight gain is usually associated with and serves as hallmark of congestion during acute HF exacerbation. Treatment guidelines include the recommendation to optimize euvolemic status. Congestion encompasses indicators of volume overload: orthopnea, jugular venous distention, congested on chest X-ray, a gain of greater than or equal to two pounds in the previous week, edema, and the need to increase diuretic dosing at a visit. Patients that were not congested had an $87 \%$ survival, compared $41 \%$ of patients with major congestion. As such, this leads to higher re-admission rate [31].

On the contrary, in a retrospective analysis of the randomized clinical trial -Diuretic Optimization Strategy Evaluation in Acute Heart Failure (DOSEAHF) studied markers of decongestion at 72 hours: weight loss and net fluid loss level and found no correlation between either weight gain or re-admission. Thus, fluid retention has not been reliable as a sole entity in detecting early decompensation. Increased appetite may result in weight gain and can mimic fluid retention [32].

Anemia can be prevalent in the SNFs population due to dental problems, decreased appetite and poor nutrition. Anemia makes HF worse and has been shown to impact readmission rates. Readmission of patients with heart failure and anemia as secondary diagnoses, were significant $(\mathrm{p}<0.001)$ in SNFs population. Patients with heart failure and anemia had increased LOS (7.3 versus 5.1 days) and unplanned readmission $(\mathrm{p}<0.001)$. In a retrospective study of 127 elderly SNF population with HF, anemia (71\%) led to higher readmission [28]. This suggests reduced hemoglobin may merely be a marker for the epiphenomena of advanced heart failure. Furthermore, anemia was an independent predictor of re-admission and mortality in heart failure patients with reduced left ventricular dysfunction [33].

Length of stay (LOS) and number of ED visits have been shown to provide additional information regarding re-admission. A significant proportion of patients are discharged to SNFs [34]. In a retrospective study of more than 10,000 patients admitted with HF, $30 \%$ were discharged to SNFs [34]. This may be in an attempt to decrease length of stay in the hospital. In a simulated study of the relationship between LOS and readmission within 7 days and 30 days, it was reported that if there was a 1 day increase in LOS, reductions in readmission rates could be estimated in the $1 \%$ to $8 \%$ range for HF patients [35]. Therefore increasing LOS for some patients may be a means of decreasing readmission and improving quality of care.

However, other investigators found no associations between LOS and readmission [36]. Patients and hospitals with longer length of stay showed reduced readmission rate in $\mathrm{HF}$ patients [37]. Alternatively, individuals hospitalized for heart failure, had no increase in 30-day re-hospitalization when LOS was decreased by a day [38].

Functional status/ADL. Frailty, mobility, disability, and impaired ADLs status are associated with readmissions. According to Heart Failure Society of America, functional capacity is defined in terms of ambulation. Patients who present with exacerbation of heart failure are typically dyspneic and fatigued on presentation. Consequently, patients will often limit their physical activity in order to compensate for their worsening heart failure symptoms. Anderson found that individuals with HF who require assistance with ADLs were significantly more likely to be readmitted for heart failure within 60 days [12].

Elevated Serum biomarkers, such as Brain Natriuretic peptide (BNP) have been associated with increasing rate of readmissions [14]. Reduction in NT-proBNP was significantly associated with symptom relief $(\mathrm{r}=$ $0.13, \mathrm{P}=0.04)$ in a retrospective analysis of 308 elderly HF subjects, suggesting that positive relationship exist between commonly used markers of decongestion and patient reported symptom relief and less chance of readmission [39]. Similarly, early dyspnea relief in 2984 patients studied was associated with lower BNP value with resultant reduced readmissions [14].

Hypotension is associated with readmission and patients that are hypotensive, had previous SNF's stay, or decompensated during the night, and tend to have higher re-admissions [26]. Systolic BP of less than 115 was associated with $15 \%$ risk of readmission and death [25]. The hypotensive episodes in the SNFs population may be attributable to administration of Guideline Directed Medical Therapy, such as the ACEi and ARBs that often prescribed lowers the blood pressure. Fifty three percent cases of HF following MI, and HF following uncontrolled hypertension were deemed avoidable [23]. This findings led to the move toward further research into identification of high risk patients and avoidable readmission.

Comorbidities increase the risk of re-admissions in older HF patients. Associated diagnosis of anemia, hypertension, and diabetes confer higher risk and are likely indicator of disease progression and are 
predictors of re-admissions and mortality $[6,10,26,28,40]$. Comorbidity has been shown as a predictor of unplanned readmission [41]. In addition, comorbidities were associated with increasing readmission in $>80$ year old within 30 days with comorbidities odds ratio $=2.6 ; 95 \%$ CI, 1.5-4.7 [42] Comorbidities were found to be frequently associated with 7 and 30 day readmission of SNF patients [43]. Older HF patients from SNFs residents who had acute episode leading to readmission had higher level of comorbidities with acute HF [26].

The purpose of this study was to identify patient factors affecting re-admission of elderly patients with Heart failure within 30 days who were discharged to SNFs. The identified factors may predict patient related mediators of early re-admissions and may assist with the development of interventions that may reduce 30 days re-admissions.

\section{Methodology}

\section{Design}

A retrospective chart review was conducted to examine patterns and factors affecting readmission of SNF patients with HF. Eligible patient records were identified from a tertiary institution's Electronic Medical Record as unplanned or emergent, general or direct re-admissions as from 2012 to 2014 until the 128 subjects obtained.

\section{Sample/Patient selection}

Medical records of 128 consecutive elderly patients, admitted to an 800-bed tertiary institution were reviewed. Inclusion criteria included the following: Patients with a heart failure diagnosis (DRG 428) and subsequently discharged to NH or SNF. All patients had to be insured by Medicare or Medicaid to increase generalizability. SNF patients admitted through the ED, admitted electively or from the outpatient settings were included. SNF patients transferred from other hospitals were excluded from the chart review.

Medical records of eligible patients were reviewed to confirm diagnosis of HF using Framingham criteria and DRG coding. Data collection was performed using a standardized data extraction tool. Follow-up data includes information on subsequent hospital readmission, survival status, heart transplantation, and visits to the emergency department using the electronic medical record. All patients' data were reviewed for 180-days following discharge and to account for 30day readmissions and readmission patterns over 6 months.

\section{Inclusion criteria}

Age 65years or >

NYHA II-III
Heart Failure Diagnosis DRG 428

Patients discharged to SNF

Exclusion criteria

Age $<65$ years

Hyperthyroidism

Terminal illnesses

NYHA I, IV

Severe cognitive impairment

\section{Severe COPD}

CKD on dialysis

Severe Aortic Stenosis

Patients discharged home

Major psychiatry illness

Sample size including power analysis

Approximately 128 patients that met the inclusion criteria as described above were included in the study. A power analysis indicated that a sample size of 109 patients was sufficient (power .80) with an alpha of .05 to detect statistically significant results. Oversampling of patients was done to allow for exploratory statistical analysis. One hundred and nine $(\mathrm{n}=109)$ patients with $15 \%(n=19)$ oversampling included for a total $N=128$. Multiple regression analysis included eight independent variables of interest identified from the theoretical framework and previous studies.

\section{Operational definition of independent variables and dependent variables}

Patient associated factors including dyspnea, increasing age renal problems, fluid status/weight, anemia, LOS, ADLs, BNP, hypotension, and comorbidities age were identified as predictors and were continuous variables. The dependent variables were clinical events, such as re-admission within 30 days or ED admissions.

\section{Data collection procedures}

Eligible patients were identified using the inclusion criteria as described above. Data was collected on Heart Failure patients, 65 years or older, that were discharged from a tertiary institution in the Northeastern US to Nursing Homes and Skilled Nursing facilities from 2012 to 2014 until the target subject number of 128 was reached. The data on readmission was collected on any of the patients who were readmitted for any reason from the electronic medical record.

Demographic data: age, sex, race etc. will be obtained

Symptoms: Dyspnea, wt/fluid status, swelling/edema, fatigue, 
Diagnosis: Systolic or diastolic Heart failure, NYHA classification,

Vital signs: Blood pressure, HR, height, weight

Medications: ACEi, BB, Mineralocorticoids

Blood tests results: BUN/Cr, BNP, sodium, hematocrit, HBa1c (if applicable)

Number of re-admissions

Length of Stay

\section{Analysis}

The data collected and analyzed in this study included socio-demographic information, medical conditions, symptoms, guideline directed medical therapy (GDMT), functional capacity, and significant test and clinical findings upon assessment. The sample size of participants in the study was one twenty eight, $(\mathrm{n}=128)$ elderly subjects with heart failure (HF) readmission from SNF. The dependent variable was hospital readmission. All patients studied were patients discharged to SNFs, of age 65 years or older, with primary or secondary diagnosis of $\mathrm{CHF}$, and experiencing multiple chronic conditions. Hospital readmission was defined as any readmission within 30 days after hospital discharge to SNF's.

Descriptive statistics (frequencies and percentages for categorical data; mean \pm standard deviation and median for continuous data) and univariate analyses using the chi-square test or Fisher's exact test, as deemed appropriate, for categorical variables and the
Mann-Whitney test for continuous data was used to compare patients readmitted within 30 days vs. those who were not readmitted within 30 days. Those factors that appeared to be associated with readmission in the univariate analysis $(\mathrm{p}<0.10)$ were included in the selection process for a multivariate logistic regression model. Best subsets selection was used as a screening method to identify the best set of predictor variables for the logistic regression model.

The analysis of LOS was accomplished by applying standard methods of survival analysis, i.e., computing the Kaplan-Meier product limit curves, where the data were stratified by readmission within 30 days (Yes vs. No). The groups were compared using the log-rank test. The median rates for each group were obtained from the Kaplan-Meier/Product-Limit Estimates and their corresponding 95\% confidence intervals were computed, using Greenwood's formula to calculate the standard error. Unless otherwise specified, a result was considered statistically significant at the $\mathrm{p}<0.05$ level of significance.

\section{Results}

The datasets included patients with HF with mean age 83.46 years. Twenty three (18\%) of the patients had at least one re-admission.

The baseline social-demographic distribution N (\%) showed the patients were distributed as 60 (46.9.9\%) males and $68(53.1 \%)$ females with the majority (56 $(43.8 \%))$ in the age category greater than 85 years (Table 1).

Table 1: Baseline Distribution of the social-demographic information of the Study Population.

\begin{tabular}{|c|c|c|c|c|c|}
\hline Variable & $\mathbf{N}$ & $\%$ & Variable & $\mathbf{N}$ & $\%$ \\
\hline Gender & & & Ethnicity & & \\
\hline Male & 60 & 46.9 & Hispanic or Latino & 1 & 0.8 \\
\hline Female & 68 & 53.1 & Not Hispanic or non-Latino & 127 & 99.2 \\
\hline Race & & & Marital status & & \\
\hline Caucasian & 122 & 95.3 & Married & 50 & 39.1 \\
\hline Black or African-American & 2 & 1.6 & Single & 21 & 16.4 \\
\hline Asian & 2 & 1.6 & Widowed & 52 & 40.6 \\
\hline Unknown or not reported & 2 & 1.6 & Divorced & 4 & 3.1 \\
\hline \multicolumn{6}{|l|}{ Age Category } \\
\hline $65-75$ y & 22 & 17.2 & & & \\
\hline $76-85$ y & 50 & 39.1 & & & \\
\hline
\end{tabular}


Given the location of the study hospital, 122 patients (95.3\%) were Caucasian, 2 (1.6\%) were black/African American, and 2 (1.6\%) were Asian. Fifty two (40.6\%) of the patients were widowed and $50(39.1 \%)$ were married. Subjects that were studied had a mean heart rate of 79.18 , mean systolic blood pressure of 131.5 $\mathrm{mmHg}$ and mean diastolic blood pressure of 68.6 $\mathrm{mmHg}$. The mean brain natriuretic protein (BNP) was 1174.89 , mean BUN was 40.12 with mean creatinine of 1.60. The average hematocrit was 33.99, left ventricular ejection fraction (LVEF) was 47.22, and differences in weight measurements were 9.91. The mean length of days (LOS) for the first admission was
9.22 days while the LOS for the readmission was 10.91days.

The associated medical conditions and co-morbidities showed $70(54.7 \%)$ patients had history of renal disease and 89 (69.5\%) patients had history of hypertension. Most of the patients had no history of CAD 67(52.3\%), no history of angina 120 (93.8\%) and no history of MI $108(84.4 \%)$. In spite of patients residing at SNF, only $4(3.1 \%)$ had additional meds administered prior to admission to relieve symptoms (Table 2).

\begin{tabular}{|c|c|c|c|c|c|}
\hline \multicolumn{3}{|l|}{ Ambulation } & \multicolumn{3}{|l|}{ Assisted devices } \\
\hline Not ambulatory & 59 & 46.1 & Not using assistive devices & 90 & 70.3 \\
\hline Ambulatory & 69 & 53.9 & uses assistive devices & 38 & 29.7 \\
\hline Needs assistance with ADL & 121 & 94.5 & Not independent with bathing & 123 & 96.1 \\
\hline $\begin{array}{l}\text { Does not require assistance with ADLs } \\
\text { (independent) }\end{array}$ & 7 & 5.5 & independent with bathing & 5 & 3.9 \\
\hline Independent with toileting & 21 & 16.4 & & & \\
\hline
\end{tabular}

The subjects experienced high level of functional limitations. About 107 (83.6\%) required assistance with toileting, $123(96.1 \%)$ required assistance with bathing and $121(94.5 \%)$ required assistance with ADLs. Despite the high level of limitations, only 38 (29.7\%) used assistive devices (Table 3).

Table 3: Guideline Directed Medical Therapy (GDMT).

\begin{tabular}{|l|l|l|l|l|l|}
\hline Variable & N & Variable & N & \\
\hline Beta blocker & & Mineralocorticoids & 112 & 87.5 \\
\hline Not on beta blocker & 39 & 30.5 & Not on mineralocorticoid & 16 & 12.5 \\
\hline On betablocker & 89 & 69.5 & On mineralocorticoids & & \\
\hline Diuretics & & & Angiotensin Receptor Blockers (ARBs) & 110 & 85.9 \\
\hline Not on diuretics & 51 & 39.8 & Not on ARBs & 18 & 14.1 \\
\hline On diuretics & 77 & 60.2 & On ARBs & & \\
\hline Digoxin & & & Nitrates & 105 & 82 \\
\hline Not on Digoxin & 113 & 88.3 & Not on nitrates & 23 & 18 \\
\hline On Digoxin & 15 & 11.7 & On Nitrates & \\
\hline
\end{tabular}




\begin{tabular}{|l|l|l|l|l|l|}
\hline Hydralazine & & & Angiotensing Converting Enzyme inhibitor (ACEi) & & \\
\hline Not on Hydralazine & 106 & 82.8 & Not on ACEi & 91 & 71.1 \\
\hline On Hydralazine & 22 & 17.2 & On ACEi & 36 & 28.1 \\
\hline & & & Missing & 1 & 0.8 \\
\hline
\end{tabular}

Fifteen candidate variables, creatinine, weight difference, CKD, Angina, Arrhythmia, VHD, Tobacco, ADL, independent in bathing, independent in the toilet, S3 Heart sounds present, HJR, AF, Nitrates, and Hydralazine, were included in the selection process for the multivariate logistic regression as they were potential risk factors associated with "readmission within 30 days". Since there were only 23 readmissions within 30 days, based on the rule-ofthumb that for every one predictor variable included in a multivariate model there needs to be 10 "events", our final model included only 2 predictor variables. Creatinine and ADLs were included in the final model as this subset of predictors was found to be the best for prediction of "readmission within 30 days" (Figure 1).

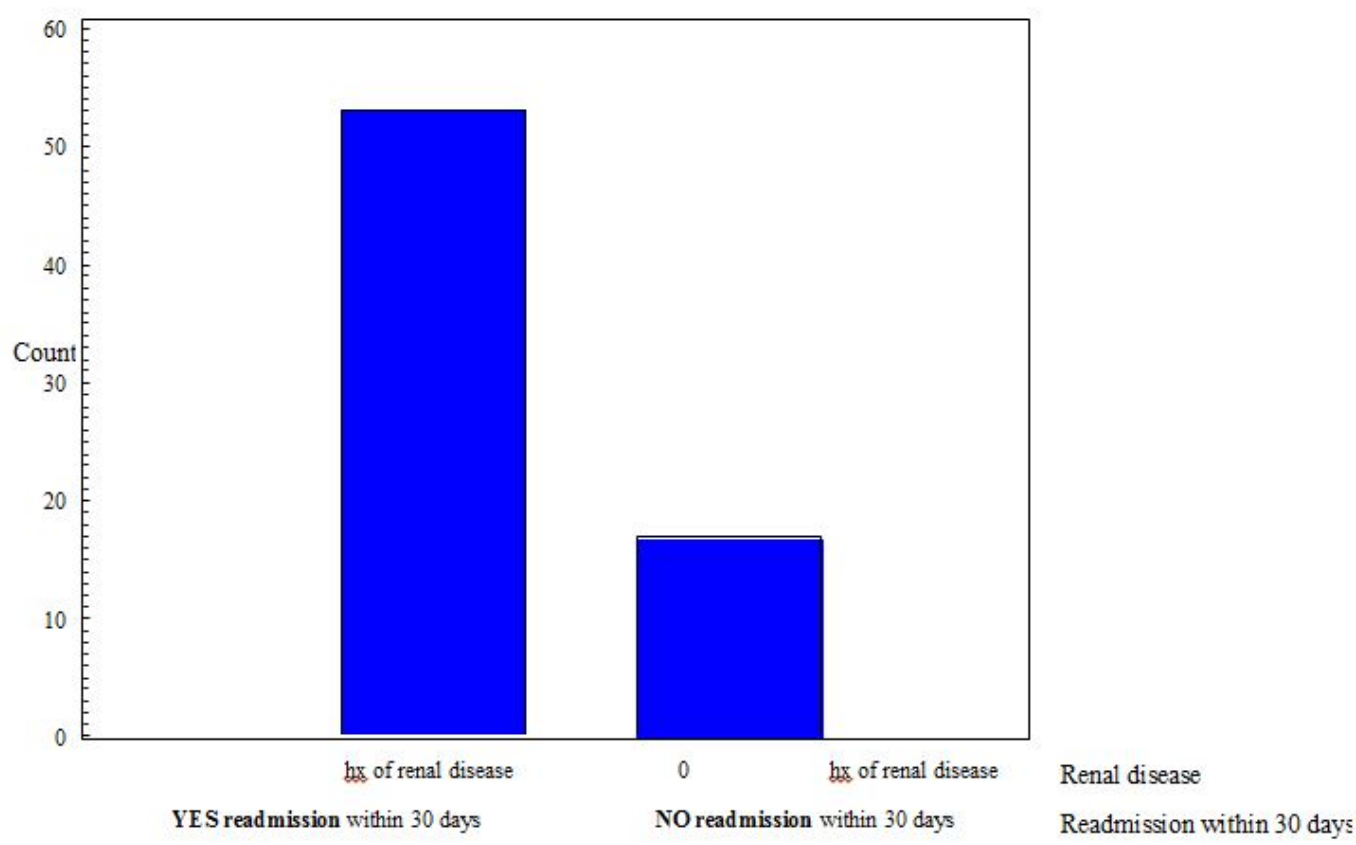

Renal disease

Figure 1: Renal disease.

Creatinine $(\mathrm{p}<0.0087)$ and ADLs $(\mathrm{p}<0.0077)$ were both significantly associated with readmission within 30 days in the final logistic regression model. Every 1unit increase in creatinine was associated with an $87 \%$ increase in the odds of being readmitted within 30 days $(\mathrm{OR}=1.87)$. Those patients who required assistance with ADLs were over 9 times more likely to be readmitted within 30 days $(\mathrm{OR}=9.25)$ as compared to patients who are independent (Figure 2). 


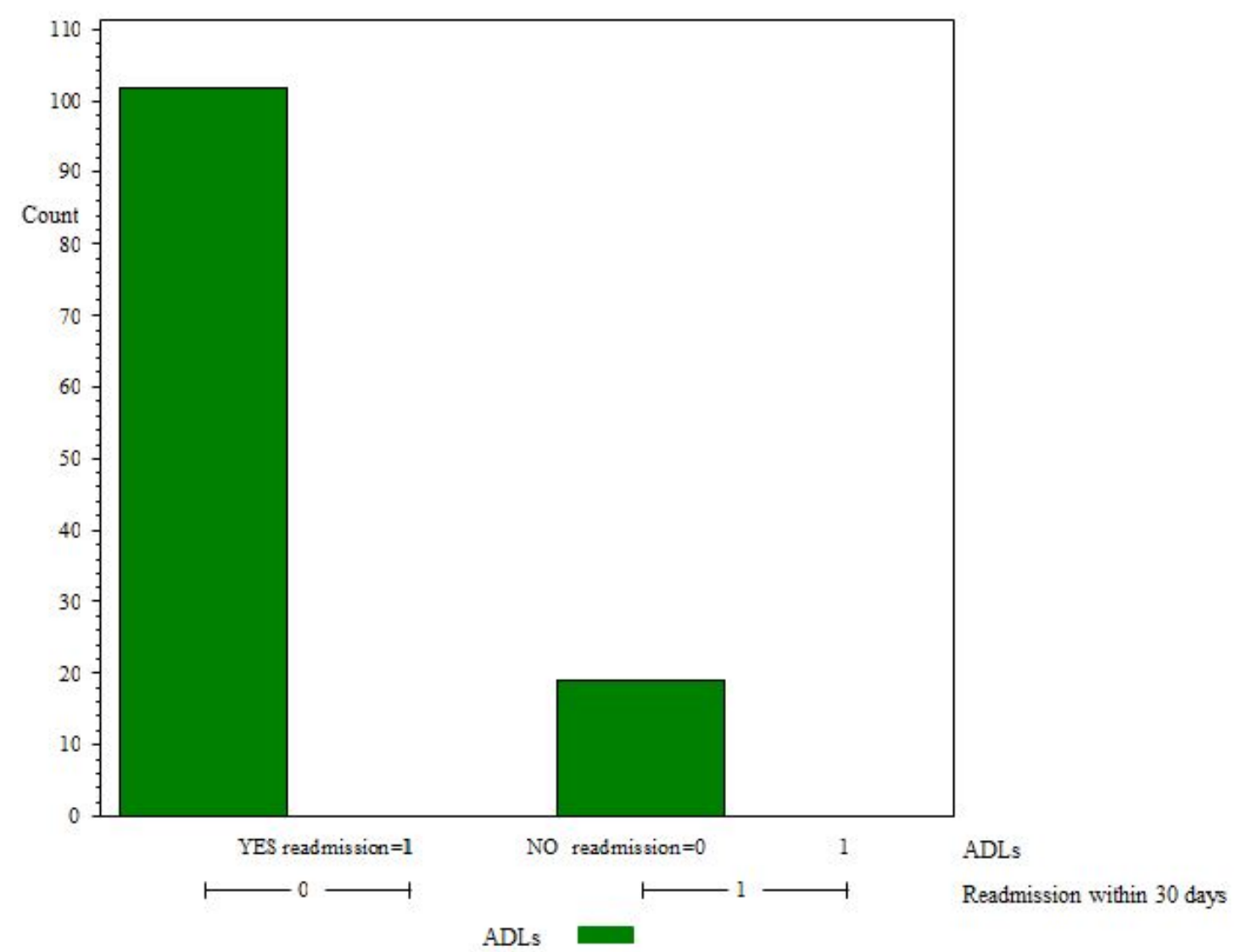

Figure 2: ADLs.

\section{Discussion}

There were fifteen variables that were significant to readmissions within 30 days in the SNFs population studied. However, there were only 23 readmissions within 30 days; thus, our final model included only 2 predictor variables. Creatinine and ADLs were included in the final model as this subset of predictors was found to be the best for prediction of "readmission within 30 days".

Increased creatinine revealed that many of the patients who were readmitted into the hospital had decreased renal functioning. (Although this has been shown as a predictor of readmissions and GDMT has been shown to improve morbidity, a significant proportion of the studied population were not on therapy. About $72 \%$ of the populations studied were not on Angiotensin Converting enzyme inhibitor (ACEi), 86\% were not on angiotensin receptor blocker (ARBs), 30\% were not on betablocker, and $88 \%$ were not on mineralocorticoids. These are medications that can help improve HF condition and has been shown to decrease mortality and reduce re-admissions yet most of the patients were not placed on these medications [3].

However, 60 percent of the patients studied were receiving symptom relief using diuretics. Diuretics have not been shown to reduce readmissions, decrease mortality and are not evidence based because research did not show that diuretics reduce morbidity, mortality, and they do not change the outcome [3]. The lack of use of the evidence based medications such as betablockers and Angiotensin Receptor Inhibitors (ACEi) in the population studied might be a contributing factor to re-admission. The evidence based therapies were not prescribed for $60 \%$ of the population studied.

Findings of decreased renal function are significant in the SNFs population. Worsening renal function was reported as the strongest indicator for readmissions in the SNFs population $[10,14,24-30]$. As such, assessment of intolerance of neuro-hormonal antagonists and its antecedent hypotension with worsening renal function is of utmost importance. Patients with chronic kidney disease represented a unique sub-population in this cohort. They constitute challenges to maintenance of fluid volume status since the management goal is focused on preservation of the remaining renal function.

The second finding, ambulatory status, showed that patients with a decreased functional status were more likely to be readmitted. The study findings suggest that patients requiring assistance with ADLs were more likely to be re-admitted. The older the HF patients, the 
more likely the need for assistance, and the higher readmission rate. Patients with limitations in their day to day functioning as evidenced by impairment in their ability to perform activities of daily living are more likely to be re-admitted. This finding supports the recent study that shows patients with $\mathrm{HF}$ who require assistance with ADLs were significantly more likely to be readmitted for heart failure within 60 days [12]. The SNFs may need to invest more in the improvement and maintenance of functionality of the patients.

The heterogeneity of the patient factors examined may be attributable to demographic changes. However, subtle changes in status need to be recognized in view of the multiple co-morbidities in the elderly population with HF. Investigators may find better discrimination for predicting re-admission in patients that are discharged after heart failure hospitalizations by including cognitive impairment and care-seeking behaviors. It may be interesting to find out if certain predictors are paramount to gender or race. There is clearly a need for better discrimination of which predictors of readmission is of significance in certain cohorts of HF patients.

There is no consensus as to what the predictors of HF are at the present time. Complex interaction of many patient factors may lead to readmission. Further investigation and refinement of the patient factors affecting readmissions may lead to consensus.

\section{Study Limitation}

Study population was limited to retrospective study using electronic medical record (EMR) from a large sub urban teaching hospital. This may limit the generalizability of the results. The study sample was small, it was racially and ethnically limited to primarily caucasian and representative of the population in the community. The distribution of participants on some variables was uneven and would have led to biased findings. From a statistical perspective, and in order to build a more complex multivariate model, a larger sample size would be necessary. As a result, future studies should be conducted with larger and more diverse samples to prevent biased findings. Also, there were only a very small group of patients readmitted from the SNF so it was very difficult to find the predictors.

\section{Recommendations}

Patients with chest pain and inconclusive ECG findings should undergo Echocardiographic examinations with strain analysis as early as possible, both to facilitate early exclusion of significant coronary disease and thus lead to early discharge and overall cost savings, also less exposure to radiation and ionizing contrast material of MPI and Coronary angiography. We recommended this study to be extended in a large scale to validate our observations.

\section{Conclusion}

The study findings provide insight into some of the patient factors that affect readmission rates in the population of older adult from SNFs in a sub-urban region in the North Eastern part of USA. Findings from this study add strength to previous findings that poor renal function and ADL were among several factors that affect readmission rates. Since the study finding suggest that worsening renal function contributes to readmission, close monitoring of renal function at discharge and post-discharge period may be useful in reducing risk of hospital readmission. Health care professionals should emphasize close monitoring of renal function. Effects of limitations in the performance of ADL on readmissions should be studied to find out if improvement in functional abilities of HF patients in SNF may reduce readmissions. Future study with larger samples is indicated to verify the current findings for generalization.

\section{Executive Summary}

Background: Despite guideline-driven pharmacological therapies and careful transitional care, the rates of preventable hospital readmission of heart failure patients and associated costs remain unacceptably high in the SNF populations. Transfer to SNF is one strategy to limit hospitalizations. As such, $25 \%$ of patients are still symptomatic at time of discharge.

Purpose: The objective of this study is to identify patient factors affecting re-admissions of HF patients residing in SNF within 30days.

Methods: A retrospective electronic chart review was completed on patients $>65$ years with HF who were admitted into large medical center between 2012 and 2014. Descriptive statistics and univariate analyses using the chi-square test or Fisher's exact test for categorical variables and the Mann-Whitney test for continuous data was used to compare patients readmitted within 30 days $v s$. those who were not readmitted within 30 days. Significant factors associated with readmission in the univariate analysis ( $p$ $<0.10$ ) were included for a multivariate logistic regression model. The analysis of LOS was accomplished by applying standard 
methods of survival analysis, i.e., computing the Kaplan-Meier product limit curves, where the data were stratified by readmission within 30 days (Yes vs. No). No data were considered 'censored'. The groups were compared using the log-rank test. The median rates for each group were obtained from the Kaplan-Meier/Product-Limit Estimates and their corresponding 95\% confidence intervals were computed, using Greenwood's formula to calculate the standard error. Unless otherwise specified, a result was considered statistically significant at the $p<0.05$ level of significance.

Results: Fifteen variables: creatinine, weight difference, CKD, Angina, Arrhythmia, VHD, Tobacco, ADL, independent in bathing, independent in the toilet, S3 Heart sounds present, HJR, AF, Nitrates, and Hydralazine, were identified for the multivariate logistic regression as potential risk factors associated with "readmission within 30 days". Based on 23 readmissions within 30 days, our final model included only 2 predictor variables. Creatinine and ADLs were included in the final model as this subset of predictors was found to be the best for prediction of "readmission within 30 days". Creatinine $(p<0.0087)$ and ADLs $(p<0.0077)$ were both significantly associated with readmission within 30 days in the final logistic regression model. Every 1-unit increase in creatinine was associated with an $87 \%$ increase in the odds of being readmitted within 30 days $(O R=1.87$ ). Those patients who require assistance with ADLs were over 9 times more likely to be readmitted within 30 days $(O R=9.25)$ as compared to patients who are independent. The final model as this subset of predictors was found to be the best for prediction of "readmission within 30 days". Creatinine $(p<0.0087)$ and ADLs $(p<0.0077)$ were both significantly associated with readmission within 30 days in the final logistic regression model. Every 1 -unit increase in creatinine was associated with an $87 \%$ increase in the odds of being readmitted within 30 days $(O R=1.87)$. Those patients who require assistance with $A D L s$ were over 9 times more likely to be readmitted within 30 days $(O R=9.25)$ as compared to patients who are independent.

\section{References}

1. Jencks SF, Williams MV, Coleman EA. Rehospitalizations among patients in the Medicare fee-for-service program. $N$. Engl. J. Med. 360(14), 1418-1428 (2009).

2. Heidenreich PA, Albert NM, Allen LA, et al. Forecasting the impact of heart failure in the United States: a policy statement from the American Heart Association. Circ. Heart. Fail. 6(3), 606-619 (2013).

3. Yancy CW, Jessup M, Bozkurt B, et al. 2013 ACCF/AHA guideline for the management of heart failure: a report of the American College of Cardiology Foundation/American Heart Association Task Force on Practice Guidelines. J. Am. Coll. Cardiol. 62(16), e147-239 (2013).

4. Walsh EG, Wiener JM, Haber S, Bragg A, Freiman M, Ouslander JG. Potentially avoidable hospitalizations of dually eligible Medicare and Medicaid beneficiaries from nursing facility and Home and Community-Based Services waiver programs. J. Am. Geriatr. Soc. 60(5), 821-829 (2012).

5. Desai AS, Stevenson LW. Rehospitalization for heart failure: predict or prevent? Circulation. 126(4), 501-506 (2012).

6. Ouslander JG, Perloe M, Givens JH, Kluge L, Rutland T, Lamb G. Reducing potentially avoidable hospitalizations of nursing home residents: results of a pilot quality improvement project. J. Am. Med. Dir. Assoc. 10(9), 644-652 (2009).

7. Unroe KT, Greiner MA, Colon-Emeric C, Peterson ED, Curtis LH. Associations between published quality ratings of skilled nursing facilities and outcomes of medicare beneficiaries with heart failure. J. Am. Med. Dir. Assoc. 13(2), e181-186 (2012).

8. Jung M, Yeh AY, Pressler SJ. Heart failure and skilled nursing facilities: review of the literature. J. Card. Fail. 18(11), 854-871 (2012).

9. Krumholz HM, Chen YT, Wang Y, Vaccarino V, Radford MJ, Horwitz RI. Predictors of readmission among elderly survivors of admission with heart failure. Am. Heart. J. 139(1), 72-77 (2000).

10. Allen LA, Hernandez AF, Peterson ED, et al. Discharge to a skilled nursing facility and subsequent clinical outcomes among older patients hospitalized for heart failure. Circ. Heart. Fail. 4(3), 293-300 (2011).

11. Altice NF, Madigan EA. Factors associated with delayed careseeking in hospitalized patients with heart failure. Heart. Lung. 41(3), 244-254 (2012).

12. Anderson KM. Discharge clinical characteristics and 60-day readmission in patients hospitalized with heart failure. J. Cardiovasc. Nurs. 29(3), 232-241 (2014).

13. Fonarow GC, Committee, Adhere Scientific Advisory. The Acute Decompensated Heart Failure National Registry
(ADHERE): opportunities to improve care of patients hospitalized with acute decompensated heart failure. Rev. Cardiovasc. Med. 4, S21-30 (2003).

14. Mentz RJ, Hernandez AF, Stebbins A, et al. Predictors of early dyspnoea relief in acute heart failure and the association with 30-day outcomes: findings from ASCEND-HF. Eur. J. Heart. Fail. 15(4), 456-464 (2013).

15. Mentz RJ, Kjeldsen K, Rossi GP, et al. Decongestion in acute heart failure. Eur. J. Heart. Fail. 16(5), 471-482 (2014).

16. Mentz RJ, Mi X, Sharma PP, et al. Relation of dyspnea severity on admission for acute heart failure with outcomes and costs. Am. J. Cardiol. 115(1), 75-81 (2015).

17. Friedman MM, Quinn JR. Heart failure patients' time, symptoms, and actions before a hospital admission. J. Cardiovasc. Nurs. 23(6), 506-512 (2008).

18. Jurgens CY, Lee CS, Reitano JM, Riegel B. Heart failure symptom monitoring and response training. Heart. Lung. 6, 56 (2013).

19. Muus KJ, Knudson A, Klug MG, Gokun J, Sarrazin M, Kaboli P. Effect of post-discharge follow-up care on readmissions among US veterans with congestive heart failure: a rural-urban comparison. Rural. Remote. Health. 10(2), 1447 (2010).

20. Boxer RS, Dolansky MA, Frantz MA, Prosser R, Hitch JA, Pina IL. The Bridge Project: improving heart failure care in skilled nursing facilities. J. Am. Med. Dir. Assoc. 13(1), e81-87 (2012).

21. Dolansky MA, Xu F, Zullo M, Shishehbor M, Moore SM, Rimm AA. Post-acute care services received by older adults following a cardiac event: a population-based analysis. $J$. Cardiovasc. Nurs. 25(4), 342-349 (2010).

22. Hammill BG, Curtis LH, Fonarow GC, et al. Incremental value of clinical data beyond claims data in predicting 30-day outcomes after heart failure hospitalization. Circ. Cardiovasc. Qual. Outcomes. 4(1), 60-67 (2011).

23. Vinson JM, Rich MW, Sperry JC, Shah AS, McNamara T. Early readmission of elderly patients with congestive heart failure. J. Am. Geriatr. Soc. 38(12), 1290-1295 (1990).

24. Allen LA, Smoyer TKE, Smith DM, Wilson KL, Agodoa I. Rates and predictors of 30-day readmission among commercially insured and Medicaid-enrolled patients hospitalized with systolic heart failure. Circ. Heart. Fail. 5(6), 672-679 (2012).

25. Fonorow GC. Importance of in-hospital initiation of evidence-based medical therapies for heart failure: taking advantage of the teachable moment. Congest. Heart. Fail. 11(3), 153-154 (2005).

26. Hutt E, Frederickson E, Ecord M, Kramer AM. Associations among processes and outcomes of care for Medicare nursing 
home residents with acute heart failure. J. Am. Med. Dir. Assoc. 4(4), 195-199 (2003).

27. Lazzarini V, Mentz RJ, Fiuzat M, Metra M, O'Connor CM. Heart failure in elderly patients: distinctive features and unresolved issues. Eur. J. Heart. Fail. 15(7), 717-723 (2013).

28. Tamhane U, Voytas J, Aboufakher R, Maddens M. Do hemoglobin and creatinine clearance affect hospital readmission rates from a skilled nursing facility heart failure rehabilitation unit? J. Am. Med. Dir. Assoc. 9(3), 194-198 (2008).

29. Wang SP, Lin LC, Lee CM, Wu SC. Effectiveness of a self-care program in improving symptom distress and quality of life in congestive heart failure patients: a preliminary study. J. Nurs. Res. 19(4), 257-266 (2011).

30. Wang Y, Tuomilehto J, Jousilahti P, et al. Lifestyle factors in relation to heart failure among Finnish men and women. Circ. Heart. Fail. 4(5), 607-612 (2011).

31. Lucas C, Johnson W, Hamilton MA, et al. Freedom from congestion predicts good survival despite previous class IV symptoms of heart failure. Am. Heart. J. 140(6), 840-847 (2000).

32. Kociol RD, Liang L, Hernandez AF, et al. Are we targeting the right metric for heart failure? Comparison of hospital 30-day readmission rates and total episode of care inpatient days. Am. Heart. J. 165(6), 987-994 (2013).

33. Al-Ahmad A, Rand WM, Manjunath G, et al. Reduced kidney function and anemia as risk factors for mortality in patients with left ventricular dysfunction. J. Am. Coll. Cardiol. 38(4), 955-962 (2001).

34. Hutt E, Elder SJ, Fish R, Min SJ. Regional variation in mortality and subsequent hospitalization of nursing residents with heart failure. J. Am. Med. Dir. Assoc. 12(8), 595-601 (2011).

35. Carey K. Measuring the Hospital Length of Stay/Readmission Cost Trade-Off under a Bundled Payment Mechanism. Health. Econ. 4, 620, (2014).

36. Kaboli PJ, Go JT, Hockenberry J, et al. Associations between reduced hospital length of stay and 30-day readmission rate and mortality: 14-year experience in 129 Veterans Affairs hospitals. Ann. Intern. Med. 157(12), 837-845 (2012).

37. Eapen ZJ, Reed SD, Li Y, et al. Do countries or hospitals with longer hospital stays for acute heart failure have lower readmission rates?: Findings from ASCEND-HF. Circ. Heart. Fail. 6(4), 727-732 (2013).

38. Unruh MA, Trivedi AN, Grabowski DC, Mor V. Does reducing length of stay increase rehospitalization of medicare fee-for-service beneficiaries discharged to skilled nursing facilities? J. Am. Geriatr. Soc. 61(9), 1443-1448 (2013).

39. Kociol RD, McNulty SE, Hernandez AF, et al. Markers of decongestion, dyspnea relief, and clinical outcomes among patients hospitalized with acute heart failure. Circ. Heart. Fail. 6(2), 240-245 (2013).

40. Chen J, Ross JS, Carlson MD, et al. Skilled nursing facility referral and hospital readmission rates after heart failure or myocardial infarction. Am. J. Med. 125(1), e101-109 (2012).

41. Hallerbach M, Francoeur A, Pomerantz SC. Patterns and predictors of early hospital readmission in patients with congestive heart failure. Am. J. Med. Qual. 23(1), 18-23 (2008).

42. Marcantonio ER, McKean S, Goldfinger M, et al. Factors associated with unplanned hospital readmission among patients 65 years of age and older in a Medicare managed care plan. Am. J. Med. 107(1), 13-17 (1999).

43. Ouslander JG, Berenson RA. Reducing unnecessary hospitalizations of nursing home residents. N. Engl. J. Med. 365(13), 1165-1167 (2011). 